\title{
Evaluation of an educational program for the caregivers of persons diagnosed with a malignant glioma
}

\author{
by Rosemary Cashman, Lori J. Bernstein, \\ Denise Bilodeau, Geoff Bovett, Barbara Jackson, \\ Masoud Yousefi, Anca Prica, James Perry
}

\section{Abstract}

Background: Caring for a loved one with a malignant glioma can be a formidable responsibility. The guarded prognosis, side effects of treatments, and changes in brain function, personality and behaviour pose unique challenges in care provision by family members. It is rare that institutions provide educational programs for caregivers.

Purpose: To evaluate the impact of providing information in an educational program to caregivers of patients diagnosed with a malignant glioma.

Methods: A structured educational program for caregivers of brain tumour patients was developed based upon multidisciplinary expert opinion and caregiver feedback. Twenty-four caregiver participants were enrolled in the program. Knowledge was assessed before, immediately following, and four to six weeks following the program. Open-ended questions were used to explore the caregivers' experiences, as well as additional benefits derived from the program.

Results: Knowledge scores on testing immediately after the program and four to six weeks following the program were statistically significantly improved from baseline testing, although there was a decline in scores four to six weeks after the program. These findings demonstrate effective knowledge transfer (recall of the information) immediately after the education program and four to six weeks later. Specific qualitative and quantitative data serve as a basis for understanding caregivers' needs and experiences.

\section{Background}

Primary malignant gliomas account for approximately two per cent of the cases of adults diagnosed with cancer, although the incidence of these tumours is increasing, particularly among the elderly (Deorah, Lynch, Sibenaller \& Ryken, 2006). Recent advances in treatment have led to modest improvements in survival for those diagnosed with the most lethal and common of these brain tumours, glioblastoma multiforme, but curative treatments remain elusive (Stupp et al., 2005). Average survival for patients with glioblastoma is still just over one year. Patients undergoing treatment for malignant brain tumours are subject to treatment side effects as well as changes produced by the tumour. These include seizures, changes in personality, mood and behaviour, and impairment of vision, hearing and mobility. Because of the specific challenges associated with this diagnosis, caregivers of brain tumour patients may bear a particularly heavy care burden and experience significant distress (Keir et al., 2006; Sherwood et al., 2006; Villejo \& Meyers, 1991).

In reviewing the major concerns of caregivers of cancer patients in general, Northouse and Peters-Golden (1993) categorized their needs into three general areas. These include dealing with the fear and uncertainty of the diagnosis (Borneman et al., 2003; Gotay, 1984; Northouse \& Peters-Golden, 1993) which may be greater for family members than for patients themselves (Matthews, 2003), helping partners to manage the emotional repercussions of the diagnosis (Strang, Strang \& Ternestedt, 2001; Toseland, Blanchard \& McCallion, 1995), and managing the disruptions in the family's usual routine that are engendered by the illness (Given et al., 2005; Laizner, Yost, Barg \& McCorkle 1993; Oberst \& James, 1985). Laizner et al. (1993) also reported that some family caregivers felt the need to be hyper vigilant of their ill family member.
In a study examining cancer caregivers, their perceived coping efficacy and social support were related to decreased caregiver strain and depression, as well as improved patient functional status (Schumacher, Dodd \& Paul, 1995) Provision of information helps caregivers to manage anxiety and reduces uncertainty associated with the cancer diagnosis and its treatment (Blanchard, Ruckdeschel \& Albrecht, 1997). Information can help caregivers assist their loved ones to make treatment decisions and may lead to enhanced patient care (Adams, 1991). Support and information from health care providers may facilitate the psychological preparedness of caregivers and, in turn, promote better family adjustment to changing roles, relationships, and the emotional challenges of serious illness (Archbold et al., 1990; Scherbring, 2002). Pasacreta, Barg, Nuamah and McCorkle (2000) found that a psycho-educational program for cancer caregivers promoted participants' adjustment to caregiving tasks even when those tasks increased over time. Furthermore, these caregivers' perceptions of their own health also improved over time. By contrast, Schulz and Beach (1999) found that spousal caregivers who experienced strain in caring for family members with cardiovascular disease had a significantly greater risk of mortality themselves.

A study by Wong et al. (2002) reported that caregivers' interest in information was generally greater and included a wider range of topics than the cancer patients under their care. Study participants identified the management of pain and fatigue and information about accessing home care services as the most important areas of interest. They also reported potential impediments to attending such an event, including parking costs and the lack of a substitute caregiver.

We conducted a needs assessment of 20 caregivers seen at a Canadian neuro-oncology clinic to determine whether an educational program for caregivers of patients with malignant gliomas would be of interest, what information was desired, and whether there were obstacles to attendance. The results indicated a strong interest in attending an educational program, with only two caregivers citing distance from the cancer centre as a barrier to their participation. Content areas that were

At the time of writing, Rosemary Cashman, RN, MA, MSc(A), ACNP, was an Advanced Practice Nurse, Neuro-oncology, Crolla Family Brain Tumour Research Unit, Sunnybrook Health Sciences Centre, Toronto, ON.

Lori J. Bernstein, PhD, Psychologist, Crolla Family Brain Tumour Research Unit, Sunnybrook Health Sciences Centre, Toronto, ON.

Denise Bilodeau, BSW, MSW, RSW, Social Worker, Sunnybrook Health Sciences Centre, Toronto, ON.

Geoff Bovett, MD, Palliative Physician, Sunnybrook Health Sciences Centre, Toronto, $O N$.

Barbara Jackson, BScOT, OTReg(Ont), Occupational Therapist, Sunnybrook Health Sciences Centre, Toronto, ON.

Masoud Yousefi, MSc, Statistician, Brain Research Centre, University of British Columbia, Vancouver, BC.

Anca Prica, MD, University of Toronto School of Medicine, Toronto, $O N$.

James Perry, MD, Neuro-oncologist, Crolla Family Brain Tumour Research Unit, Sunnybrook Health Sciences Centre, Toronto, ON.

Corresponding author: Rosemary Cashman, $R N, M A, M S c(A)$, ACNP, BC Cancer Agency, Vancouver Centre, 600 West 10th Avenue, Vancouver, BC V5Z 4E6 Phone 604-877-6072, Fax 604-877-6215 
rated most important included information about treatments, management of symptoms, providing emotional support to patients, and dealing with patients' changes in memory and thinking. Availability of a substitute caregiver, childcare provision, and assistance with parking costs were mentioned as factors that would facilitate attendance.

\section{Purpose}

The primary purpose of the study was to determine whether an educational program for the caregivers of brain tumour patients is an effective means of transmitting clear health information and improving specific knowledge for this group. We were also interested in learning about other potential benefits of such a program. Finally, we sought a better understanding of the particular nature of caregivers' needs and experiences.

\section{Methods}

Development of the educational program: Based on the results of our needs assessment, and on the expert opinion of the investigators about which information was most critical for caregivers of people with brain tumours, our program content was determined. It included brain tumour biology and treatments, symptom and side effect management, safety in the home, the role of palliative care, brain behaviour relationships, understanding and coping with cognitive changes, and obtaining psychosocial support. Program structure included introduction of participants, PowerPoint presentations by investigators (a neuro-oncologist, advanced practice nurse, palliative physician, occupational therapist, social worker and neuropsychologist), Q \& A periods, group discussion, and informal interaction of participants at scheduled refreshment breaks.

\begin{tabular}{|l|l|}
\hline \multicolumn{2}{|l|}{ Table One. Program evaluation } \\
\hline $\begin{array}{l}\text { Response set } \\
1=\text { not at all, } 2 \text { = a bit, } 3=\text { somewhat, } 4 \text { = quite a bit, 5 = }\end{array}$ \\
\hline Statement & $\begin{array}{l}\text { Mean } \\
\text { Score }\end{array}$ \\
\hline Wellspring was a good place to hold the program. & 4.92 \\
\hline The material presented was easy for me to understand. & 4.83 \\
\hline The hand-outs were helpful. & 4.50 \\
\hline I have a clearer sense of resources available to me. & 4.35 \\
\hline $\begin{array}{l}\text { The program helped me to better understand about the } \\
\text { care and treatment of people with brain tumours. }\end{array}$ & 4.35 \\
\hline $\begin{array}{l}\text { The presentations covered material that was } \\
\text { important to me. }\end{array}$ & 4.25 \\
\hline $\begin{array}{l}\text { The timing of the program was about as convenient } \\
\text { as it could have been for me. }\end{array}$ & 4.17 \\
\hline $\begin{array}{l}\text { The question periods allowed me to ask } \\
\text { some of my specific concerns. }\end{array}$ & 3.83 \\
\hline $\begin{array}{l}\text { I feel more capable of providing better } \\
\text { care for my loved one. }\end{array}$ & 3.75 \\
\hline I feel more confident in my abilities as a caregiver. \\
\hline \begin{tabular}{l} 
I feel less anxious about my role as a caregiver. \\
\hline $\begin{array}{l}\text { Not having to pay for parking made it possible } \\
\text { for me to attend. }\end{array}$
\end{tabular} & 3.67 \\
\hline $\begin{array}{l}\text { Having care provided for my children made it } \\
\text { possible for me to attend. }\end{array}$ & 1.07 \\
\hline \begin{tabular}{l} 
Having care provided for my loved one made it \\
\hline
\end{tabular} & 1.0 \\
\hline
\end{tabular}

Setting: Each educational program session took place for two consecutive half-days at a Wellspring facility on the hospital campus This facility provides emotional and psychological support, free of charge, to individuals and families living with cancer. The cost of parking was covered by a research grant. Childcare was available at the hospital playroom and supervision for patients was available in a separate room at the facility. Refreshments and lunch were provided.

Development of the test-questionnaire: Knowledge was assessed through a four-alternative multiple-choice test consisting of 31 questions related to the program content. The first drafts of test questions were developed by dividing content into specific areas. Investigators independently developed questions related to their areas of clinical expertise, and a composite test of all questions was shared with the entire research team. Changes were made by the team for maximum face validity and clarity. The first 10 caregivers encountered at regularly scheduled clinic visits during a single week were asked to take the test to ensure that questions were clearly worded. There were no refusals, and no further changes to the test were required.

Results ranged from nine to 23 correct responses out of 31 with a mean of 18.5 , median of 20 , standard deviation of 4.20 , and standard error of the mean of 1.33. Based on these data, and on the investigators' belief that an increase of five correct responses $(16 \%)$ from pre- to post-test would constitute a significant increase in knowledge, sample size was determined. Twenty-one participants were required for an alpha of .01 and a power of 90 (beta.10). In an effort to keep the group small so as to better address specific individual informational needs, we offered the program on two separate occasions, accepting 13 participants in the first two-day session and 11 in the second two-day session, for a total of 24 participants, thus allowing for dropouts. For study participants, the test was first completed after participants provided their informed consent for enrolment in the program (baseline knowledge), again at the end of each program session (post-study intervention), and finally four to six weeks following the program to determine whether knowledge had been retained.

Target population: Study participation was offered to the caregivers of patients with primary brain tumours at a neuro-oncology clinic. There were no exclusion criteria and we allowed more than one caregiver of any given patient to participate.

Other instruments: Questions were developed by the investigators to assess the extent to which participants felt the program met their needs (Table One). Each item was evaluated on a five-point scale from $1=$ not at all to $5=$ very. Open-ended questions were also developed by the research team to better understand the needs and experiences of caregivers. Written responses to these questions were obtained from participants at the end of each two-day session. These were reviewed verbally with participants by investigators to clarify and amplify responses and to correct any misunderstandings. Demographic information was collected at registration.

Ethical considerations: The protocol was reviewed and approved by the research ethics board. Informed consent was obtained from participants before any study procedure was conducted. The patient was also obliged to provide written informed consent for his or her caregiver to participate in the study and to share specific details about the patient's care and condition with the group. Assessments were conducted in the course of regularly scheduled visits (e.g., registration and pre-test), as a part of the educational program session, or by telephone or fax at the four- to six-week follow-up assessment.

Procedure: Caregivers were approached during the course of regularly scheduled clinic visits to identify those interested in participating in an educational program. Registration and pre-tests 
were conducted prior to the first program session, either in clinic or on the first day of the program, according to the participant's preference. At the end of each day, participants completed a test of multiple choice questions related to the material covered on that day. At the end of the two-day session, evaluation of the program and interviews about the nature of the caregiving experience were conducted.

\section{Results}

Sample characteristics. Eighty per cent (20/24) of study participants were female, well-educated (21/24 had at least some university) relatives of malignant glioma patients. One-third was between the ages of 46 and $55(8 / 24)$ and most identified themselves as the primary caregiver of the patient (21/24). Spouses accounted for 12 of the 24 participants, the largest group of caregivers (nine wives, three husbands).

Data analysis. Multiple choice test questions: Mean correct response per cent scores were calculated at each time of test administration. Data were analyzed for statistical significance by employing the Friedman post-hoc nonparametric analysis, corrected for multiple comparisons. A two-sided $\mathrm{p}$ value was calculated, and statistical significance was accepted at the 0.05 level. From baseline to first postintervention testing, scores were statistically significantly improved. Despite a drop in mean scores from the first post-intervention testing, scores remained statistically significantly higher than at baseline at the second post-intervention testing (four to six weeks after the educational program), indicating retention of knowledge (Figure One).

Program evaluation: The questionnaires evaluating the effectiveness of the program were scored in such a way that endorsement of positive responses and nonendorsement of negative responses were assigned higher values. The questions were averaged into one composite score ranging from one to five. The program was very favourably evaluated by participants, with mean scores over four for statements related to program content, location, timing and outcomes related to awareness of resources (Table One). Six participants (25\%) said they would have liked to participate in such a program earlier in their caregiving experience. Seventeen $(71 \%)$ noted that it was particularly beneficial to share their experiences with other caregivers. Among the specific benefits noted were feeling "we are not unique or alone in what we go through," and learning that "others are going through similar difficulties, or worse." Sixteen per cent appreciated the opportunity to meet with professional staff in an informal manner and ask specific questions. Two participants indicated that one of the most important outcomes of the program was the validation they received for their caregiving roles: "You have acknowledged us as primary caregivers" and (as a result of the program interaction) "I feel that I have done some things correctly." Assistance with childcare, patient care and parking fees were not significant factors in program participation for this group of caregivers.

Caregiver experience: Qualitative analysis of open-ended questions employed the constant comparative method in which responses were analyzed for emerging concepts. Concepts were grouped together to form categories, and categories were grouped to form themes. Each statement within each category was compared to other categories to ensure that data reflected similar ideas. As new data were collected they were assigned to existing categories or to new categories as necessary. Dominant themes emerging from the responses to openended questions relate to specific challenges of the caregiving role and factors that promote coping with these challenges. These include:

Living with uncertainty: Participants described ever-changing circumstances that left them feeling fearful, anxious and apprehensive, even despite available support.

"We're barely used to the most recent change in (her brother's) abilities and then there will be another change (...) and we (as a family) have to change routines, hopes, plans."
"I fear that I won't be able to cope with what lies ahead (for my mother) even with the help that's offered."

Providing emotional support to patients despite personal feelings of distress: Participants described lives of intense pressure juggling many roles, battling fatigue, facing down their own sadness and fears for the future and, yet, being called upon to provide a supportive and reassuring outlook to patients.

"...he wants everything to be "normal" at a time when every aspect of our lives has been turned upside down."

"(The biggest challenge for me $)$ is being patient with him when the tumour is talking. When he gets agitated and is not making sense, it's hard for me to stay calm and get (him) to calm down."

“...we should be hopeful and encouraging to the patient... it's often hard to believe what you're saying is true, and in my brother's case, he's still planning 10 years down the road and it's hard not to tell him that there may not be that 10 years."

Developing caregiver abilities: Participants discussed the stress engendered by their lack of confidence in their caregiving abilities. They struggled to balance a patient's desire for independence - "I cannot say no to him about going alone in a fishing boat" - with the need to protect them from harm, and frequently "second-guessed" their own caregiving decisions. The wife of a patient reported that even armed with sufficient knowledge she was "unsure that [she had] the necessary guts and abilities" to care for her husband.

Meeting never-ending demands: For many caregivers, their loved one's illness obliged them to take on many new roles and responsibilities besides that of caregiver. Parents with children were particularly burdened with "balancing all tasks required - caring for a toddler, cleaning, cooking, being effective at work, etc." As a result, they struggled to find a respite from their stressful responsibilities.

"I feel like I always have to tend to his needs and ignore mine... (it's important for me to) fit time in for myself so I don't get run down and (so I) can give better care."

The ceaseless demands also created a sense of isolation that further compromised caregivers' health and well-being.

"You can't have too much knowledge": The participants in this program attended with the expectation that they would learn about caring for their loved ones. Not surprisingly, all study participants mentioned that they felt they coped with the challenges of caregiving better when armed with information.

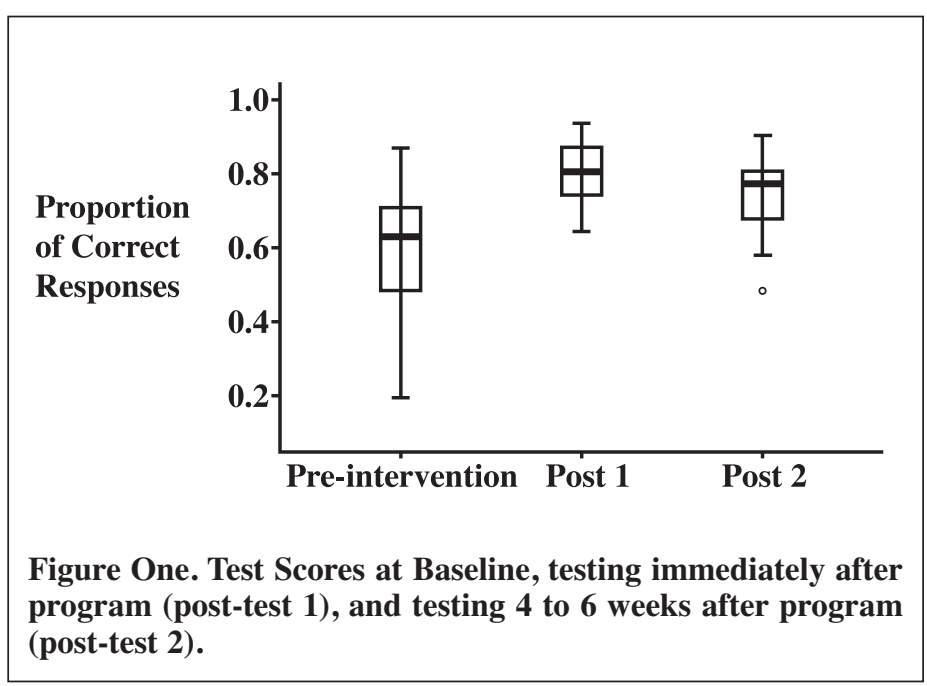


The value of normal times: A day without distractions and disruptions to routines, when the patient's condition was stable, and when families could momentarily return to a life that resembled the one they shared before the diagnosis "and practise some denial" was a balm to caregivers and patients alike.

\section{Discussion}

This educational program resulted in the acquisition of knowledge for the program participants and addressed their stated desire for more information. It was also successful, although to a lesser degree, in promoting confidence in caregiving abilities and reducing anxiety. The program provided an opportunity for participants to share their stories, normalize threatening experiences and, sometimes, even compare their own situations favourably to the difficulties faced by other participants who might be younger, have small children, or be caring for sicker patients. An important outcome articulated by two participants was a sense of validation of the importance of their caregiving role. Many participants emphasized the need for such support earlier in the experience caring for a loved one.

The transfer of knowledge does not, however, necessarily result in enhanced caregiving skills or psychological adjustment to the experience of caring for a seriously ill family member. In addition to the intense physical demands of care, caregivers of individuals with malignant brain tumours must cope with the ongoing psychological threat of an incurable disease that causes their loved ones to "fade away" in every sense, through changes in behaviour and personality and decrements in cognitive and physical abilities. Caregivers must balance their knowledge and fears about the terminal nature of the patient's illness with their own desire to be hopeful and to convey a sense of optimism to the ill person. Programs aimed at promoting caregiving competence must, therefore, go beyond providing information and address the psychological needs of caregivers of brain tumour patients in order to promote a sense of mastery, integrity and coherence in the experience of caring for a loved one who may be fundamentally changed by the tumour and treatment, and who will, ultimately, die of the disease.

\section{Limitations of the study}

Among the limitations of this study is the small, homogenous sample of participants. And, as is often the case, the individuals who were perhaps most in need of support did not participate in this program. This study offered information in a supportive setting that favoured interaction of participants. As such, it attracted those participants who were educated and relatively comfortable talking about their experiences. Caregivers who were not accustomed to processing and retaining new information, did not speak English well, or were not interested in sharing feelings in a group, either because of distress or other factors, did not attend this program. The caregivers in this study were able to attend the program without significant concerns about childcare, patient care or parking costs, suggesting that they may have either benefited from some support already or cared for loved ones who were not precariously ill. Participants may also have overstated the positive effects of the intervention because of a desire to please the health care professionals caring for their loved ones.

\section{Implications for practice and future research}

Twenty-one of the caregivers in this study (87\%) cared for individuals who died of their disease within four weeks to 12 months of program participation. Given et al. (2005) noted increased burden and depression in caregivers who were female, nonspousal, employed caregivers of cancer patients with multiple symptoms, such as malignant glioma patients. A large proportion of the participants in our study were spouses of the patients, a relationship that may be associated with a better caregiving response (Chapman \& Pepler, 1998; Given et al., 2005). In addition, these study participants demonstrated openness to problem-solving through their participation in an educational program. This characteristic may have helped them in the bereavement period as well (Schut, Stroebe, van den Bout \& de Keijser, 1997). Similar educational programs might be relevant for the caregivers of other medically vulnerable individuals in the last months of their lives.

Brain tumours turn the lives of patients and their families upside down. Caregivers need assistance in acquiring a wealth of new information related to treatments and side effects. They also need to learn to manage their own emotional distress, meet the demands of many competing roles and relationships, and live with pervasive and unrelenting uncertainty. The research literature suggests factors that promote successful caregiving, and factors that pose a threat to these abilities.

An extensive body of literature demonstrates a range of improved outcomes for caregivers who employ active coping strategies, such as problem-solving and information-seeking, rather than emotionfocused strategies to achieve a sense of self-efficacy and control. These include improved caregiver quality of life, life satisfaction and positive mood, and lower levels of caregiver depression and strain (Haley, Levine, Brown \& Bartolucci, 1987; Keefe et al., 2002; Wallhagen, 1992). Nurses can facilitate caregiver efforts to analyze and solve problems, obtain information, make decisions and reduce anxiety (Heim, Valach \& Schaffner, 1997). Caregivers who are encouraged to mobilize support through family and friends may find emotional sustenance, as well as assistance with the instrumental tasks of caregiving, work, and household demands. Identification of formal support systems such as hospice and visiting nurses may also provide respite (Sherwood, Given, Doorenbos \& Given, 2004). Caregivers can be assisted to reframe negative events, set achievable goals, and interact with those who promote and validate their strengths. The positive emotions that result from successful adaptations may act as a buffer against distress. A randomized controlled trial allocating participant caregivers to either a psychoeducational program or standard palliative care found that the experimental program had a significant impact on the perceived rewards of participating caregivers when compared to the standard care participants (Hudson, Aranda \& Hayman-White, 2005). Nursing care approaches and educational programs that help caregivers to find meaning in the illness and in their caregiving experience might also allow them to identify previously unacknowledged abilities and unexpected benefits of the experience (Germino, Fife \& Funk, 1995; Sherwood et al., 2004).

Not all families are distressed or unsuccessful in adapting to the demands of caring for a family member with cancer; research indicates that a subgroup of approximately $20 \%$ of families experience very high levels of distress (Edwards \& Clarke, 2004). Nurses must be aware of the risk factors for poor adjustment to caregiving, including multiple demands on the caregiver (Northouse et al., 2002), uncertainty about how to support family members in deteriorating health (Barg et al., 1998) and age. Although younger patients and their families may experience greater distress and disruption in the life cycle because of a cancer diagnosis, older individuals may be obliged to deal with cancer and associated caregiving against a background of pre-existing functional difficulties and comorbidities (Northouse, 1994). Future research might investigate the needs of non-Caucasian, non-traditional, and lower socioeconomic status families in meeting the challenges of cancer, especially a cancer that is as uniquely threatening as a malignant glioma. Formal educational programs will not address all the needs of all caregivers but, by introducing appropriate informational support and validation, even in the brief context of a clinic visit or phone call, nurses may provide a lifeline to caregivers facing critical challenges. 
Through the thoughtful assessment of individuals and families living with a malignant glioma, nurses can help to obviate obstacles to competent caregiving, emphasize the inherent strengths and abilities that these individuals and families possess, and develop individualized approaches to support them through this experience.

\section{References}

Adams, M. (1991). Information and education across the phases of cancer care. Seminars in Oncology Nursing, 7(2), 105-11.

Archbold, P.G., Stewart, B.J., Greenlick, M.R., \& Harvath, T. (1990). Mutuality and preparedness as predictors of caregiver role strain. Research in Nursing and Health, 13(6), 375-384.

Barg, F.K., Pasacreta, J.V., Nuamah, I., Robinson, K.D., Angeletti, K., Yasko, J., et al. (1998). A description of a psychoeducational intervention for the family caregivers of patients with cancer. Journal of Family Nursing, 4, 394-413.

Blanchard, C.G., Ruckdeschel, J.C., \& Albrecht, T.L. (1997). The crisis of cancer: Psychological impact on family caregivers. Oncology, 11(2), 189-94.

Borneman, T., Chu, D.Z.J., Wagman, L., Ferrell, B., Juarez, G., McCahill, L.E., et al. (2003). Concerns of family caregivers of patients with cancer facing palliative surgery for advanced malignancies. Oncology Nursing Forum, 30(6), 997-1005.

Deorah, S., Lynch, C.F., Sibenaller, Z.A., \& Ryken, T.C. (2006). Trends in brain cancer incidence and survival in the United States: Surveillance, epidemiology, and end results program, 1973 to 2001. Neurosurg Focus, 20(4) E3.

Chapman, K., \& Pepler, C. (1998). Coping, hope and anticipatory grief in family members in palliative care. Cancer Nursing, 21, 226-34.

Edwards, B., \& Clarke, V. (2004). The psychological impact of a cancer diagnosis on families: The influence of family functioning and patients' illness characteristics on depression and anxiety. Psycho-oncology 13, 562-76.

Germino, B.B., Fife, B.L., \& Funk, S.G. (1995). Cancer and the partner relationship: What is its meaning? Seminars in Oncology Nursing, 11, 43-50.

Given, B., Wyatt, G., Given, C., Gift, A., Sherwood, P., DeVoss, D., et al. (2005). Burden and depression among caregivers of patients with cancer at the end-of-life. Oncology Nursing Forum, 31(6), 1105-17.

Gotay, C.C. (1984). The experience of cancer during early and advanced stages: The views of patients and their mates. Social Science and Medicine, 18, 605-613.

Haley, W.E., Levine, E.G., Brown, S.L., \& Bartolucci, A.A. (1987). Stress, appraisal, coping, and social support as predictors of adaptational outcome among dementia caregivers. Psychology and Aging, 2(4), 323-330.

Heim, E., Valach, L., \& Schaffner, L. (1997). Coping and psychosocial adjustment: Longitudinal effects over time and stages in breast cancer. Psychosomatic Medicine, 59, 408-18.

Hudson, P.L., Aranda, S., \& Hayman-White, K. (2005). A psychoeducational intervention for family caregivers receiving palliative care: A randomized controlled trial. Journal of Pain and Symptom Management, 30(4), 329-41.

Keefe, F.J., Ahles, T.A., Porter, L.S., Sutton, L.M., McBride, C.M., Pope, M.S., et al. (2002). The self-efficacy of family caregivers for helping cancer patients manage pain at end-of-life. Pain 103(1-2),157-62.

Keir, S.T., Guill, A.B., Carter, K.E., Booles, L.C., Gonzales, L., \& Friedman, H.S. (2006). Differential levels of stress in caregivers of brain tumor patients - Observations from a pilot study. Support Care Cancer [Epub ahead of print].

Laizner, A., Yost, K.M.S., Barg, F.K., \& McCorkle, R. (1993). Needs of family caregivers of persons with cancer: A review. Seminars in Oncology Nursing, 9(2), 114-20.

Matthews, B.A. (2003). Role and gender differences in cancer-related distress: A comparison of survivor and caregiver self-reports. Oncology Nursing Forum, 30, 493-99.

\section{Acknowledgement}

The authors wish to thank Dr. Margaret Fitch for reviewing the manuscript. They also acknowledge the support of a Practice-Based Research Award and the Crolla Family Brain Tumour Research Unit at Sunnybrook Health Sciences Centre in Toronto.

Northouse, L.L. (1994). Breast cancer in younger women: Effects on interpersonal and family relations. Journal of the National Cancer Institute Monographs, 16, 183-90.

Northouse, L.L., Mood, D., Kershaw, T., Schafenencker, A., Mellon, S., Walker, J., et al. (2002). Quality of life of women with recurrent breast cancer and their family members. Journal of Clinical Oncology, 20, 4050-64.

Northouse, L.L., \& Peters-Golden, H. (1993). Cancer and the family: Strategies to assist spouses. Seminars in Oncology Nursing, 9(2), 74-82.

Oberst, M., \& James, R. (1985). Going home: Patient and spouse adjustment following cancer surgery. Topics in Clinical Nursing, 7, 46-57.

Pasacreta, J.V., Barg, F., Nuamah, I., \& McCorkle, R. (2000). Participant characteristics before and four months after attendance at a family caregiver cancer education program. Cancer Nursing, 23(4), 295-303.

Scherbring, M. (2002). Effect of caregiver perception of preparedness on burden in an oncology population. Oncology Nursing Forum, 29(6), 70-76.

Schumacher, K.L., Dodd, M.J., \& Paul, S.M. (1995). The stress process in family caregivers of persons receiving chemotherapy. Research in Nursing and Health, 16, 395-404.

Schulz, R., \& Beach, S.R. (1999). Caregiving as a risk factor for mortality: The Caregiver Health Effects Study. JAMA, 282, 2215-19.

Schut, H.A., Stroebe, M.S., van den Bout, J., \& de Keijser, J. (1997). Intervention for the bereaved: Gender differences in the efficacy of two counselling programmes. British Journal of Clinical Psychology, 36(Pt 1), 63-72.

Sherwood, P.R., Given, B.A., Doorenbos, A.Z., \& Given, C.W. (2004). Forgotten voices: Lessons from the bereaved caregivers of persons with a brain tumour. International Journal of Palliative Nursing, 10(2) 67-74.

Sherwood, P.R., Given, B.A., Given, C.W., Schiffman, R.F., Murman, D.L., Lovely, M., et al. (2006). Predictors of distress in caregivers of a primary malignant brain tumor. Research in Nursing and Health, 29, 105-20.

Strang, S., Strang, P., \& Ternestedt, B. (2001). Existential support in brain tumour patients and their spouses. Support Care Cancer, 9, 625-33.

Stupp, R., Mason, W.P., van den Bent, M.J., Weller, M., Fisher, B., Taphoorn, M.J.B., et al.; European Organisation for Research and Treatment of Cancer Brain Tumor and Radiotherapy Groups; National Cancer Institute of Canada Clinical Trials Group. (2005). Radiotherapy plus concomitant and adjuvant temozolomide for glioblastoma. New England Journal of Medicine, 352, 987-96.

Toseland, R.W., Blanchard, C.G., \& McCallion, P. (1995). A problemsolving intervention for caregivers of cancer patients. Social Science \& Medicine, 40, 517-28.

Villejo, L., \& Meyers, C. (1991). Brain function, learning styles, and cancer patient education. Seminars in Oncology Nursing, 7(2), 97-104.

Wallhagen, M.I. (1992). Perceived control and adaptation in elder caregivers: Development of an explanatory model. International Journal of Aging and Human Development, 36, 219-37.

Wong, R.K.S., Franssen, E., Szumacher, E., Connolly, R., Evans, M., Page, B. (2002). What do patients living with advanced cancer and their carers want to know? - A needs assessment. Supportive Care in Cancer, 10, 408-15. 